\title{
ON THE TOËPLITZ CORONA PROBLEM
}

\author{
E. Amar
}

Abstract

The aim of this note is to characterize the vectors $g=\left(g_{1}, \ldots, g_{k}\right)$ of bounded holomorphic functions in the unit ball or in the unit polydisk of $\mathbb{C}^{n}$ such that the Corona is true for them in terms of the $H^{2}$ Corona for measures on the boundary.

Let $D$ be a bounded domain in $\mathbb{C}^{n}$, the Corona problem is: given functions $g_{1}, \ldots, g_{N}$ holomorphic and bounded in $D$ such that:

$$
\forall z \in D, \quad \sum_{i=1}^{N}\left|g_{i}(z)\right|^{2} \geq \delta^{2}>0,
$$

find $f_{1}, \ldots, f_{N}$ still holomorphic and bounded in $D$ such that $\sum_{i=1}^{N} f_{i} g_{i}=1$ in $D$. This was solved for $D=\mathbb{D}$, the unit disk in $\mathbb{C}$ by L. Carleson [8] and it is still open for $n>1$ for the basic domains namely the unit ball $\mathbb{B}_{n}$ and the unit polydisk $\mathbb{D}^{n}$.

We shall link this question to a question on Toëplitz operators via the $H^{p}(\mu)$ Corona.

\section{Notations}

We are interested by the basic domains, the unit ball in $\mathbb{C}^{n}, D=\mathbb{B}_{n}$, in fact any bounded convex domain with smooth boundary $D$, or the unit polydisc $D=\mathbb{D}^{n}$.

If $D=\mathbb{D}^{n}$ we set $b D=\mathbb{T}^{n}$, the distinguished boundary; if $D$ is a bounded convex domain with smooth boundary, $b D=\partial D$ the topological boundary.

Recall that:

$$
H^{\infty}(D):=\left\{f \text { holomorphic in } D /\|f\|_{\infty}:=\sup _{z \in D}|f(z)|<\infty\right\} .
$$

2000 Mathematics Subject Classification. 32A35, 32A36, 47B35.

Key words. Corona problem, Toëplitz operators, Von Neumann Minimax theorem. 
Let $\mathcal{M}$ be the set of all probability measures on $b D$ and for $\mu \in \mathcal{M}$ and $1 \leq p<\infty$ let $H^{p}(\mu)$ be the closure in $L^{p}(\mu)$ of the holomorphic polynomials.

If $\mu \in \mathcal{M}$ and $f \in H^{\infty}(D)$ then, with the assumption that $0 \in D$, for any $r<1, f_{r}(z):=f(r z)$ is such that $f_{r} \in A(D):=H^{\infty}(D) \cap \mathcal{C}(\bar{D})$. There is a subsequence of $\left\{f_{r}, r<1\right\}$ which converges in $\left(L^{1}(\mu), L^{\infty}(\mu)\right)$ topology and uniformly on compact sets of $D$ to a $\tilde{f} \in H^{\infty}(\mu) \cap H^{\infty}(D)$. Hence for a fixed $\mu \in \mathcal{M}$ we can assume that a $f \in H^{\infty}(D)$ is in $H^{\infty}(\mu) \cap H^{\infty}(D)$.

Now suppose that the Corona problem is solvable, i.e.

$g_{1}, \ldots, g_{N} \in H^{\infty}(D)$ are such that $\exists f_{1}, \ldots, f_{N} \in H^{\infty}(D)$ with $1=$ $f_{1} g_{1}+\cdots+f_{N} g_{N}$; we have, for any polynomial $P$ :

$$
P=P f_{1} g_{1}+\cdots+P f_{N} g_{N}
$$

Let $h \in H^{p}(\mu)$. Then there is a sequence $\left\{P_{k}\right\}_{k \in \mathbb{N}}$ of polynomials such that $P_{k} \rightarrow h$ in $H^{p}(\mu)$, hence:

$$
P_{k}=\sum_{j=1}^{N} P_{k} f_{j} g_{j}
$$

but then $P_{k} f_{j} \rightarrow h_{j}$ in $H^{p}(\mu)$, because the $f_{j}$ can be seen as in $H^{\infty}(\mu) \cap$ $H^{\infty}(D)$.

So if the Corona is true then the $H^{p}(\mu)$ Corona is also true for any $\mu \in \mathcal{M}$ :

$$
C H^{p}(\mu): \forall h \in H^{p}(\mu), \quad \exists k_{1}, \ldots, k_{N} \in H^{p}(\mu) \text { s.t. } h=\sum_{j=1}^{N} g_{j} k_{j} .
$$

The aim of this paper is to show the converse.

If $f:=\left(f_{1}, \ldots, f_{N}\right)$ we set $|f|^{2}(z):=\sum_{j=1}^{N}\left|f_{j}(z)\right|^{2}$ and $\|f\|_{p}:=\||f(\cdot)|\|_{p}$, where $\|\cdot\|_{p}$ is the $L^{p}(b D, \mu)$ norm and $\|\cdot\|_{\infty}$ is the sup norm in $D$. 
Theorem 1.1. Let $D$ be a bounded convex domain $D$ containing $O$ and with a smooth boundary or the unit polydisk $\mathbb{D}^{n}$ of $\mathbb{C}^{n}, n \geq 1$. Let: $g_{1}, \ldots, g_{N} \in H^{\infty}(D)$ and $\delta>0$. The following are equivalent:

(i) There exist functions $f_{1}, \ldots, f_{N}$ in $H^{\infty}(D)$ such that $\sum_{i=1}^{N} f_{i} g_{i}=1$ and $\|f\|_{\infty}^{2} \leq \frac{1}{\delta^{2}}$.

(ii) For all measures $\mu$ on $b D$,

$\forall h \in H^{2}(\mu)$,

$$
\exists k_{1}, \ldots, k_{N} \in H^{2}(\mu) \text { s.t. } h=\sum_{j=1}^{N} g_{j} k_{j} \text { and }\|k\|_{2}^{2} \leq \frac{1}{\delta^{2}}\|h\|_{2}^{2} .
$$

Let $g_{1}, \ldots, g_{N} \in H^{\infty}(D)$ be such that

$$
\forall z \in D, \quad|g|(z)^{2}:=\sum_{j=1}^{N}\left|g_{j}(z)\right|^{2} \geq \delta^{2}>0,
$$

we already know that:

if $D=\mathbb{B}_{n}, \mu$ the Lebesgue's measure on $\partial \mathbb{B}_{n}$ and $2 \leq p<\infty$, then $C H^{p}(\mu)$ is true $[\mathbf{2}]$;

if $D=\mathbb{D}^{n}, \mu$ the Lebesgue's measure on $\mathbb{T}^{n}$ and $1 \leq p<\infty$, then $C H^{p}(\mu)$ is true [10], [11];

if $D$ is strictly pseudo-convex, $\mu$ the Lebesgue's measure on $\partial D$ and $2 \leq p<\infty$, then $C H^{p}(\mu)$ is true [5];

if $D$ is a bounded pseudo-convex domain with smooth boundary, $\mu$ the Lebesgue's measure on $\partial D$, then $C H^{2}(\mu)$ is true [4].

In the case $n=1, D=\mathbb{D}$ the unit disc in $\mathbb{C}, \mu$ the Lebesgue's measure on $\mathbb{T}$, then $C H^{2}(\mu) \Rightarrow C H^{\infty}(\mathbb{D})[\mathbf{1 2}]$, by an operator method: the commutant lifting theorem of Nagy-Foias.

This means that the Corona theorem in one variable can be proved this way, hence there is some hope to prove a general version of the Corona theorem also by this way.

\section{Proof of the theorem}

We already seen that i) $\Rightarrow$ ii); to prove that ii) $\Rightarrow$ i) we shall use the minimax theorem of Von Neuman. The minimax theorem was already used by Berndtsson $[\mathbf{6}],[\mathbf{7}]$ in order to get estimates on solutions of the $\bar{\partial}$-equation; here the situation and the method are quite different. 
We shall work with $N=2$ in order to simplify notations. Because $D$ is always convex containing 0 , we may assume by dilation that the data $g:=\left(g_{1}, g_{2}\right)$ are continuous up to the boundary, provided that the estimates do not depend on it.

Let $\Omega$ be an open set in $D$ such that $\bar{\Omega} \subset D, 0 \in \Omega$ and let, for $\epsilon>0$, $\mathcal{C}_{\epsilon}$ be:

$$
\begin{aligned}
\mathcal{C}_{\epsilon}:=\left\{\left(f=\left(f_{1}, f_{2}\right) \in A(D)^{2},\right.\right. & \text { s.t. } \left.\|1-f \cdot g\|_{\Omega} \leq \epsilon\right\} \\
& \text { where }\|f\|_{\Omega}:=\sup _{z \in \Omega}|f(z)| ;
\end{aligned}
$$

this set is clearly convex in $A(D)^{2}$. Let $\mathcal{M}$ be the set of probability measures on $b D$ and for $0<\eta \leq 1$ let $\mathcal{M}_{\eta}=\eta m+(1-\eta) \mathcal{M}$, where $m$ is the Lebesgue measure on $b D$; this is a convex weakly compact set.

Let us define $N$ as

$$
\forall f \in \mathcal{C}_{\epsilon}, \forall \mu \in \mathcal{M}_{\eta}, \quad N(f, \mu):=\|f\|_{\mu}^{2}:=\left\|f_{1}\right\|_{L^{2}(\mu)}^{2}+\left\|f_{2}\right\|_{L^{2}(\mu)}^{2} .
$$

Then $N$ is convex on $\mathcal{C}_{\epsilon}$ for $\mu$ fixed in $\mathcal{M}_{\eta}$ and concave, in fact affine, and continuous on $\mathcal{M}$ for $f$ fixed in $\mathcal{C}_{\epsilon}$, hence we can apply the minimax theorem $[\mathbf{9}]$ :

$$
\sup _{\mu \in \mathcal{M}_{\eta}} \inf _{f \in \mathcal{C}_{\epsilon}} N(f, \mu)=\inf _{f \in \mathcal{C}_{\epsilon}} \sup _{\mu \in \mathcal{M}_{\eta}} N(f, \mu) ;
$$

by (ii) with $h=1$ we have $\exists k=\left(k_{1}, k_{2}\right) \in\left(H^{2}(\mu)\right)^{2}, g \cdot k=1,\|k\|_{\mu} \leq \frac{1}{\delta}$; because $\mu=\eta m+(1-\eta) \nu$ we get $\|k\|_{m} \leq \frac{1}{\delta \sqrt{\eta}}$ hence $k \in\left(H^{2}(m)\right)^{2}$; by the very definition of $H^{2}(\mu)$ there is a sequence $f_{n} \in(A(D))^{2}$ such that $f_{n} \rightarrow k$ in $\left(H^{2}(\mu)\right)^{2}$ hence also in $\left(H^{2}(m)\right)^{2}$ hence $f_{n} \rightarrow k$ uniformly on compact sets of $D$; so for $\epsilon^{\prime} \leq \epsilon$ there is a $f \in A(D)^{2}$ with $\|f-k\|_{\Omega} \leq$ $\frac{\epsilon}{\|g\|_{\infty}}$ and $\|f-k\|_{H^{2}(\mu)} \leq \epsilon^{\prime}$. Hence we have

$$
\|1-f \cdot g\|_{\Omega}=\|k \cdot g-f \cdot g\|_{\Omega} \leq\|g\|_{\infty}\|f-k\|_{\Omega} \leq \epsilon
$$

which means that $f \in \mathcal{C}_{\epsilon}$. We deduce that the left side of $(*)$ is bounded by $\frac{1}{\delta^{2}}$ hence for any $\epsilon>0, \eta>0, \gamma>0$ there is a $f_{\epsilon, \eta, \gamma} \in \mathcal{C}_{\epsilon}$ with $\sup _{\mu \in \mathcal{M}_{\eta}} N\left(f_{\epsilon, \eta, \gamma}, \mu\right) \leq \frac{1}{\delta^{2}}+\gamma$.

Now let $a \in D$ and $\nu_{a}$ a representing measure for $a$ supported by $b D$, then we have with $\mu:=\eta m+(1-\eta) \nu_{a}$ :

$$
\left|\eta f_{\epsilon, \eta, \gamma}(0)+(1-\eta) f_{\epsilon, \eta, \gamma}(a)\right|=\left|\int f_{\epsilon, \eta, \gamma} d \mu\right| \leq \frac{1}{\delta}+\gamma
$$

and with $\mu=m$,

$$
\left|f_{\epsilon, \eta, \gamma}(0)\right|=\left|\int f_{\epsilon, \eta, \gamma} d m\right| \leq \frac{1}{\delta}+\gamma ;
$$


hence

$$
\left|f_{\epsilon, \eta, \gamma}(a)\right| \leq \frac{(1+\eta)\left(\frac{1}{\delta}+\gamma\right)}{1-\eta}
$$

because this is true for any $a \in D$ we get

$$
\left\|f_{\epsilon, \eta, \gamma}\right\|_{\infty} \leq \frac{(1+\eta)\left(\frac{1}{\delta}+\gamma\right)}{1-\eta} .
$$

Using Montel property we get that there is a $f \in\left(H^{\infty}(D)\right)^{2}$ bounded by $\frac{1}{\delta}$ and such that $g \cdot f=1$ on $\Omega$ hence, because $\Omega$ is open and $f \cdot g$ is holomorphic in $D, f \cdot g=1$ in $D$.

\section{Operator version}

We shall give an operator version of the previous result strongly inspired by $[\mathbf{3}]$, but first we need some definitions. Let $D$ be as before and $\mu \in \mathcal{M}$; for any function $f$ in $L^{\infty}(\mu)$ define the Toëplitz operator $T_{f}^{\mu}$ on the Hilbert space $H^{2}(\mu)$ by

$$
\forall g \in H^{2}(\mu), \quad T_{f}^{\mu} g:=P_{\mu}(f g),
$$

where $P_{\mu}$ is the orthogonal projection from $L^{2}(\mu)$ on $H^{2}(\mu)$. We can state:

Corollary 3.1. Let $D$ be a bounded convex domain containing 0 and with a smooth boundary or the unit polydisk $\mathbb{D}^{n}$ of $\mathbb{C}^{n}, n \geq 1$. Let: $g_{1}, \ldots, g_{N} \in H^{\infty}(D)$ and $\delta>0$. The following are equivalent:

(i) There exist functions $f_{1}, \ldots, f_{N}$ in $H^{\infty}(D)$ such that $\sum_{i=1}^{N} f_{i} g_{i}=1$ and $\|f\|_{\infty}^{2} \leq \frac{1}{\delta^{2}}$.

(ii) For all measures $\mu$ on $b D, \sum_{j=1}^{N} T_{g_{j}}^{\mu}\left(T_{g_{j}}^{\mu}\right)^{*} \geq \delta^{2} 1$.

For $D=\mathbb{D}^{2}$, this was proved in $[\mathbf{1}]$; they used a method specific to the bidisc which explicitly cannot work even for $\mathbb{D}^{3}$.

Proof: We shall prove that (ii) is equivalent to:

(iii) For all measures $\mu$ on $b D$,

$$
\forall h \in H^{2}(\mu),
$$

$$
\exists k_{1}, \ldots, k_{N} \in H^{2}(\mu) \text { s.t. } h=\sum_{j=1}^{N} g_{j} k_{j} \text { and }\|k\|_{2}^{2} \leq \frac{1}{\delta^{2}}\|h\|_{2}^{2},
$$

and then we apply the theorem to be done. 
(ii) $\Rightarrow$ (iii) (same proof as in [1] ): Let $\mu$ be a probability measure on $b D$ and set $G_{i}:=T_{g_{i}}^{\mu}$; by (ii) we get that the operator $Q:=G_{1} G_{1}^{*}+$ $\cdots+G_{N} G_{N}^{*}$ is invertible and $\left\|Q^{-1}\right\| \leq \frac{1}{\delta^{2}}$. We can define:

$$
F_{i}:=G_{i}^{*} Q^{-1}, \quad i=1, \ldots, N ;
$$

these are bounded operators on $H^{2}(\mu)$ and clearly we get:

$$
G_{1} F_{1}+\cdots+G_{N} F_{N}=1 .
$$

Now take $k_{i}=F_{i} h, k:=\left(k_{1}, \ldots, k_{N}\right)$; we have

$$
\|k\|_{2}^{2}=\left\|G_{1}^{*} Q^{-1} h\right\|^{2}+\cdots+\left\|G_{N}^{*} Q^{-1} h\right\|^{2},
$$

but

$$
\left\|G_{1}^{*} Q^{-1} h\right\|^{2}=\left\langle G_{1}^{*} Q^{-1} h, G_{1}^{*} Q^{-1} h\right\rangle=\left\langle G_{1} G_{1}^{*} Q^{-1} h, Q^{-1} h\right\rangle
$$

hence

$$
\|k\|_{2}^{2}=\left\langle h, Q^{-1} h\right\rangle \leq \frac{1}{\delta^{2}}\|h\|^{2},
$$

because $\left(G_{1} G_{1}^{*}+\cdots+G_{N} G_{N}^{*}\right) Q^{-1}=1$.

Together with equation (1) this means precisely that the $H^{2}(\mu)$ Corona is true, i.e.

(iii) $\forall h \in H^{2}(\mu)$,

$\exists k_{1}, \ldots, k_{N} \in H^{2}(\mu)$ s.t. $h=\sum_{j=1}^{N} g_{j} k_{j}$, and $\|k\|_{2}^{2} \leq \frac{1}{\delta^{2}}\|h\|^{2}$.

(iii) $\Rightarrow$ (ii): Let $\mu \in \mathcal{M}$, then by (iii) we have:

$\forall h \in H^{2}(\mu)$,

$$
\exists k_{1}, \ldots, k_{N} \in H^{2}(\mu) \text { s.t. } h=\sum_{j=1}^{N} g_{j} k_{j} \text { and }\|k\|_{2}^{2} \leq \frac{1}{\delta^{2}}\|h\|_{2}^{2},
$$

then $S_{h}:=\left\{k=\left(k_{1}, \ldots, k_{N}\right) \in\left(H^{2}(\mu)\right)^{N}: \sum_{j=1}^{N} G_{j} k_{j}=h\right\}$ is not empty and it has elements of norm less than $\frac{1}{\delta^{2}}\|h\|_{2}^{2} ; S_{0}$ is a subspace of the Hilbert space $\left(H^{2}(\mu)\right)^{N}$ hence there is a unique element $k=\left(k_{1}, \ldots, k_{N}\right)$ in $S_{h}$ which is orthogonal to $S_{0}$ and hence of minimal norm. Then we get: $\|k\|_{2}^{2} \leq \frac{1}{\delta^{2}}\|h\|_{2}^{2}$ and, defining $F_{j}$ by $F_{j} h:=k_{j}, j=1, \ldots, N$, we 
have:

$$
\begin{aligned}
& \sum_{j=1}^{N}\left\|F_{j} h\right\|_{2}^{2} \leq \frac{1}{\delta^{2}}\|h\|_{2}^{2} \\
\forall h \in H^{2}(\mu), \quad & \sum_{j=1}^{N} G_{j} F_{j} h=h .
\end{aligned}
$$

From equation (3) we get:

$$
\forall h \in H^{2}(\mu), \quad\left\langle\sum_{j=1}^{N} G_{j} F_{j} h, h\right\rangle=\|h\|_{2}^{2},
$$

hence $\forall h \in H^{2}(\mu), \sum_{j=1}^{N}\left\langle F_{j} h, G_{j}^{*} h\right\rangle=\|h\|_{2}^{2}$,

$$
\begin{aligned}
\forall h \in H^{2}(\mu), \quad\|h\|_{2}^{2} & \leq \sum_{j=1}^{N}\left\|F_{j} h\right\|\left\|G_{j}^{*} h\right\| \\
& \leq\left(\sum_{j=1}^{N}\left\|F_{j} h\right\|^{2}\right)^{1 / 2}\left(\sum_{j=1}^{N}\left\|G_{j}^{*} h\right\|^{2}\right)^{1 / 2} .
\end{aligned}
$$

Using equation (2) we get:

$$
\forall h \in H^{2}(\mu), \quad\|h\|_{2}^{2} \leq \frac{1}{\delta}\|h\|_{2}\left(\sum_{j=1}^{N}\left\|G_{j}^{*} h\right\|^{2}\right)^{1 / 2},
$$

hence $\forall h \in H^{2}(\mu), \sum_{j=1}^{N}\left\|G_{j}^{*} h\right\|^{2} \geq \delta^{2}\|h\|_{2}^{2}$ and the corollary.

\section{References}

[1] J. Agler And J. E. MCCARThy, Nevanlinna-Pick interpolation on the bidisk, J. Reine Angew. Math. 506 (1999), 191-204.

[2] E. Amar, On the corona problem, J. Geom. Anal. 1(4) (1991), 291-305.

[3] E. Amar And C. Menini, On an operator theory approach to the corona problem, Bull. London Math. Soc. 34(3) (2002), 369-373.

[4] M. Andersson, The $H^{2}$ corona problem and $\bar{\partial}_{b}$ in weakly pseudoconvex domains, Trans. Amer. Math. Soc. 342(1) (1994), 241-255. 
[5] M. Andersson and H. Carlsson, Wolff type estimates and the $H^{p}$ corona problem in strictly pseudoconvex domains, Ark. Mat. 32(2) (1994), 255-276.

[6] B. Berndtsson, $\bar{\partial}_{b}$ and Carleson type inequalities, in: "Complex analysis, II" (College Park, Md., 1985-86), Lecture Notes in Math. 1276, Springer, Berlin, 1987, pp. 42-54.

[7] B. Berndtsson, Uniform estimates with weights for the $\bar{\partial}$-equation, J. Geom. Anal. 7(2) (1997), 195-215.

[8] L. Carleson, Interpolations by bounded analytic functions and the corona problem, Ann. of Math. (2) 76 (1962), 547-559.

[9] T. W. Gamelin, "Uniform algebras", Prentice-Hall, Inc., Englewood Cliffs, N. J., 1969.

[10] S.-Y. LI, Corona problem of several complex variables, in: "The Madison Symposium on Complex Analysis" (Madison, WI, 1991), Contemp. Math. 137, Amer. Math. Soc., Providence, RI, 1992, pp. 307-328.

[11] K. C. Lin, The $H^{p}$-corona theorem for the polydisc, Trans. Amer. Math. Soc. 341(1) (1994), 371-375.

[12] C. F. Schubert, The corona theorem as an operator theorem, Proc. Amer. Math. Soc. 69(1) (1978), 73-76.

Département de Mathématiques

Université de Bordeaux I

33405 Talence

France

E-mail address: Eric.Amar@math.u-bordeaux.fr

Primera versió rebuda el 13 de desembre de 2002, darrera versió rebuda el 24 d'abril de 2003. 